Revista de Matemática: Teoría y Aplicaciones 2005 12(1 \& 2) : 97-109

CIMPA - UCR - CCSS ISSN: 1409-2433

\title{
UN MODELO MATEMÁTICO PARA LA BOLA DE FÚTBOL
}

\author{
René Escalante * Francisco Pacheco $^{\dagger}$
}

Recibido/Received: 19 Feb 2004

\begin{abstract}
Resumen
Este trabajo se refiere al análisis, estudio y resolución del problema matemático involucrado en el diseño y construcción de la cobertura de una bola, como las utilizadas en algunos deportes, con miras a lograr una bola con factores óptimos de esfericidad y redondez. El diseño de balones estructuralmente bien distribuidos ha cobrado importancia en los últimos años, sobre todo en el campo de los deportes, como el fútbol. La tendencia actual está dirigida principalmente a la búsqueda de un balón rápido que le de más dinamismo al juego. Para lograr una mayor velocidad del balón en su desplazamiento es importante que éste no sólo tenga una distribución de paneles adecuada, que le de una mayor esfericidad, sino que tenga una estructura que permita una buena distribución de la tensión existente entre los diferentes paneles.

A partir de un diseño inicial, definimos un proceso de reajuste de paneles en la cobertura del balón, que nos conducirá a obtener factores de esfericidad óptimos. Luego, a través de un proceso de "torsión", logramos agregar área a la superficie sin alterar los factores de esfericidad, resolviendo con ello el problema del faltante de área (i.e., el "Missing Area Problem", o MAP). Por último, por medio de redefinir la forma final de los paneles, proponemos estrategias de teselado que optimizan la estructura esférica de la bola.
\end{abstract}

Palabras clave: Bola, factor de esfericidad, factor de redondez, el problema del faltante de área, "twist", teselado.

\begin{abstract}
This work refers to the analysis, study and resolution of the mathematical problem involved in the design and construction of the cover of a ball, such as the ones used in sports, in order to achieve a ball with optimal roundness and sphericity factors.
\end{abstract}

*Departamento de Cómputo Científico y Estadística, División de Ciencias Físicas y Matemáticas, Universidad Simón Bolívar, Módulo Ipostel USB, Apartado 89007, Zona Postal 1086, Baruta (Sartenejas), Venezuela. E-mail: rene@cesma.usb.ve.

${ }^{\dagger}$ Two on a SeeSaw Corp., 3206 Cypress Point Dr., Missouri City, Texas 77459, U.S.A. E-mail: francisco@twoonaseesaw.com. 
The design of well distributed ball structures has grown in importance in the last years, most of all in the field of sports, such as soccer. The current trend is directed mainly towards finding a fast ball that will give more dynamism to the game. In order to achieve a greater velocity of the ball during its movement it is important that it has not only an adequate distribution of panels, that give it a greater sphericity, but also that it has a structure that allows for a good distribution of the existing tension between the panels.

Starting from an initial design, we define a process of readjustment of the panels in the ball cover, which will lead us to obtain optimal sphericity factors. Then, through a "twisting" process, we can add area to the surface without altering the sphericity factors, and solving the Missing Area Problem (or MAP). Finally, by redefining the final form of the panels, we propose tessellate strategies that will optimize the ball's spherical structure.

Keywords: A mathematical model, sphericity factor, roundness factor, the missing area problem, the "twist", tessellation.

Mathematics Subject Classification: 93A30, 00A05, 00A06.

\section{Introducción}

La historia del diseño de balones de fútbol ha sufrido cambios desde sus inicios, cuando se utilizaban coberturas de 12 paneles, que con el uso se deformaban por ser sus piezas demasiado grandes, hasta llegar al diseño actual de 32 piezas (12 pentágonos y 20 hexágonos), descrita originalmente por Arquímedes como uno de los trece poliedros semi-regulares [2], [8]. Después de la introducción de la bola "bucky" en el Mundial de México 70, tuvieron que pasar más de dos décadas para que se presentara una mejora en la esfericidad de la misma.

La idea para el balón "geo" surgió en 1992, con un diseño que modifica el de la bola "bucky" de 32 piezas (12 pentágonos y 20 hexágonos). La estrategia en el dise no de ésta bola consistió en acortar tres de los lados del hexágono [5]. A raíz de la introducción de la bola "geo", se logró mejorar la esfericidad del balón de 32 paneles, pasando de un factor, en términos porcentuales, de $99.45 \%$ a un factor de $99.94 \%$. Aunque el balón "geo" mejora la esfericidad de la bola "bucky", al momento de sumar el área de los paneles nos encontramos con que todavía existe un déficit de área (este factor pasa de un $98.64 \%$ a un 99.49\%).

En el campo de la construcción de domos geodésicos, por ejemplo, el error de esfericidad tiende a desaparecer conforme más paneles (o secciones) se agregan al diseño. El enfoque que aquí mostramos también está en conexión con los denominados problemas de teselado geodésico (i.e., "geodesic tessellations of the sphere" [1]). Estas estrategias de diseño están relacionadas con lo que se conoce como el procedimiento de embaldosamiento espacial (o "space tiling") que se define por considerar una colección de conjuntos abiertos disjuntos, cuyas clausuras cubren el plano (ver [6], [7] y [8]).

Este trabajo se refiere al análisis, estudio y resolución del problema matemático involucrado en el dise no y construcción de la cubierta de una bola, con miras a lograr un grado de esfericidad y redondez óptimos. De manera que, proponemos un modelo de bola, 
en la que las medidas de las longitudes de todas sus circunferencias, área de superficie y volumen resulten todos exactos, respecto a un radio dado $r$.

Este trabajo está organizado como sigue. En la §2, definimos parámetros y términos. Luego, en la §3, planteamos y resolvemos el problema de la esfericidad. En la §4, planteamos y resolvemos el problema de la redondez. Por último, en la $\S 5$, presentamos las conclusiones más importantes.

\section{Preliminares}

En este trabajo partimos de un diseño específico de la cobertura de la bola, y que denominaremos el diseño inicial o, simplemente, nos referiremos al mismo como el diseño original. Éste consta de cincuenta piezas, a saber, veinticuatro trapecios iguales, dieciocho cuadrados, también iguales, y ocho triángulos equiláteros (todos congruentes entre sí). Este diseño puede pensarse como una versión esférica de un cubo, en donde los ocho triángulos equiláteros corresponderían a los ocho vértices del mismo. Nos referiremos a estos triángulos como triángulos esquineros. En el centro de cada cara del cubo encontraríamos uno de los cuadrados (para un total de seis, uno por cada cara), los cuales, de ahora en adelante, denominaremos cuadrados frontales. Asimismo, a cada arista del cubo le hacemos corresponder otro cuadrado (en realidad, una de las diagonales de cada uno de estos cuadrados coincidirá con una de las aristas del cubo), para un total de doce cuadrados más; los que, de ahora en adelante, llamaremos cuadrados laterales. Por último, por cada triángulo consideraremos tres trapecios, con sus bases menores adyacentes a cada uno de los tres lados de cada uno de los triángulos equiláteros (para un total de $3 \times 8=24$ ). La Figura 1 muestra el diseño y la distribución de paneles sobre la superficie de la bola a que aquí hacemos referencia. Para obtener detalles adicionales acerca de este diseño inicial, el lector puede consultar el documento [3].
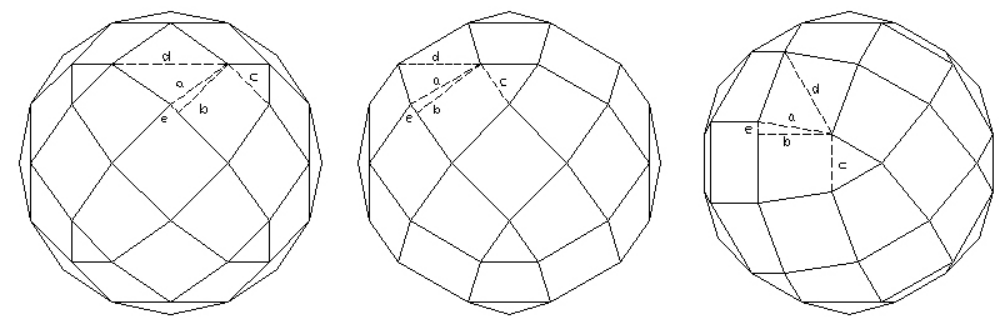

Figura 1: El diseño inicial y sus parámetros en tres vistas diferentes.

Para el desarrollo que sigue requeriremos de la siguiente notación. Denotemos por $c$ el lado de los triángulos (equiláteros) esquineros, por $d$ la diagonal de los cuadrados, por $a$ el lado de estos cuadrados (i.e., $a=\frac{d}{\sqrt{2}}$ ) y por $b$ la altura de los trapecios. Asimismo, usaremos la letra $e$ para $\frac{a-c}{2}$. Además, y para todo propósito, asumiremos que los parámetros así definidos verifican que $d>a>b>c>e$ (observemos que si $a=b \Rightarrow a=c$, y el sólido sería un cubo truncado). 
Definición 2.1 Sea r un número real mayor que cero y consideremos el conjunto de todas las circunferencias de radio $r$ que definen la superficie de una bola del mismo radio, $B(r)$. Llamaremos factor de esfericidad (SF) de la bola al cociente obtenido por dividir la longitud menor de las circunferencias asi definidas entre la de mayor longitud. Es decir,

$$
S F=\frac{\inf _{C(r) \in B(r)} l(C(r))}{\sup _{C(r) \in B(r)} l(C(r))},
$$

donde el inf y el sup se toman sobre el conjunto de todas las circunferencias de radio $r$, definidas sobre la bola (de radio $r$ ), y $l(C(r)$ ) es la longitud de la circunferencia $C(r)$.

Si bien, la Definición 2.1 es una definición de interés teórico, en la práctica, cuando deseamos medir la esfericidad de una bola, consideramos un número lo suficientemente grande de circunferencias (en general, tantas como nos permita la simetría de la bola medir), de manera que, con un cierto grado de incertidumbre, podamos asignar un número razonable al parámetro de esfericidad de la bola, $S F$. Así, por ejemplo, en el documento [3] hemos descrito en detalle cómo logramos alcanzar un grado importante de esfericidad, respecto a las longitudes de por lo menos diecisiete circunferencias, todas claramente definidas sobre la cobertura de la bola.

En este trabajo también hemos considerado diecisiete circunferencias, las cuales serán de tres tipos. Primero, las que denominaremos $C_{1}$, que medimos sobre las diagonales de los cuadrados laterales y frontales (primero un lateral, después un frontal, luego un lateral, y así sucesivamente), y que utilizaremos como patrón para nuestras mediciones posteriores. Aquí hemos fijado la longitud de estas circunferencias en $56 \mathrm{~cm}$. El segundo tipo de circunferencias por considerar son las que denominamos $C_{2}$, que medimos a lo largo de los cuadrados laterales, los triángulos esquineros y los trapecios (ver Figura ??). Por último, definimos las circunferencias $C_{3}$, que más bien son unas curvas eclípticas que definen sus trayectorias sobre los cuadrados laterales y los trapecios, de manera alternada (ver Figura ??). En este trabajo proponemos un diseño, a través del cual eliminamos el error de esfericidad, llevando este factor a un 100\%, a costa de focalizar, como veremos, la tensión de los paneles en ocho (8) puntos equidistantes sobre la superficie de la bola.

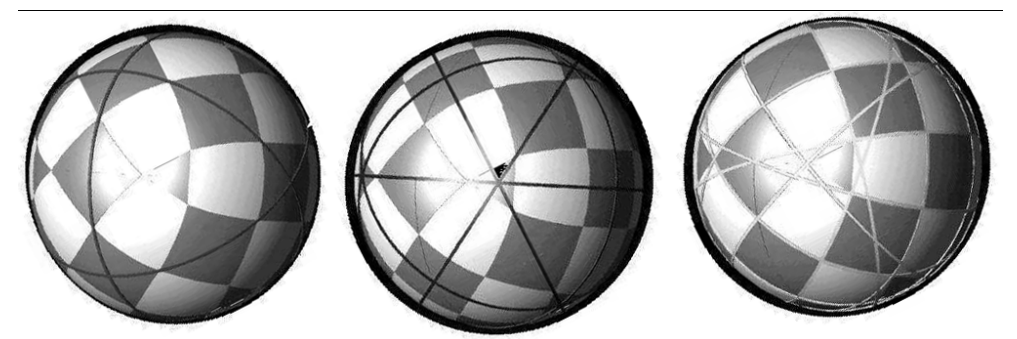

Figura 2: De izquierda a derecha: Las circunferencias $C_{1}, C_{2}$ y $C_{3}$.

Definición 2.2 Llamaremos factor de redondez (RF) a la razón obtenida de dividir la suma de las áreas de todos los paneles, en los que se ha seccionado la superficie de una 
bola, entre el área real para la cubierta de una bola de radio el correspondiente a una circunferencia de longitud fija dada (el cual es un parámetro fijo que usamos como patrón de referencia). Así, $R F=\frac{\text { area }_{p}}{\text { area }_{r}}$, donde area $_{p}$ es el área obtenida por sumar las áreas de todos los paneles que definen un modelo de bola dada, y area $a_{r}$ es el área exacta de esa bola correspondiente a un radio $r$ dado.

A través del uso de este factor contamos con una manera relativamente sencilla de medir el estado de tensión que existe entre los paneles, a la par que contamos con un parámetro que refleja la condición del problema del faltante de área (ver $\S 4.1$ ). Para los diseños conocidos, el $R F$ oscila entre un $98.5 \%$ (ver [4]) y un $99.5 \%$ (para la "geo", ver [5]).

\section{El "deltoide" y la "cometa":}

El conocido "deltoide" de la geometría tradicional, fue utilizado para configurar el sólido denominado "icositetraedro trapezoidal" o "icositetraedro deltoidal" [8], y es el poliedro dual de un "rombicuboctaedro pequeño" [8]; el mismo está compuesto por 24 paneles en forma de "deltoides". De los diseños propuestos en este trabajo, uno de ellos consiste en la distribución de 24 paneles en forma de "cometa" (i.e., el "deltoide modificado", ver Figura 3).

Definición 2.3 (La "cometa") Definimos la "cometa" como una figura compuesta por otras cinco: un trapecio (ver Figura 3), tres sectores triangulares, pertenecientes cada uno a un cuadrado de los tres que son adyacentes al trapecio (cada sector corresponde a una cuarta parte de los tres cuadrados) y, una figura triangular, adyacente a la base menor del trapecio (que corresponde a la tercera parte de cada uno de los ocho triángulos equiláteros esquineros, cada uno de los cuales está ubicado en el centro de cada octante).

En la Figura 3 podemos distinguir claramente las "cometas" en la superficie de la bola y observamos el detalle de la "cometa" en el plano, mostrando ocho vértices, a diferencia de la figura del "deltoide", en línea punteada, que tiene únicamente cuatro.

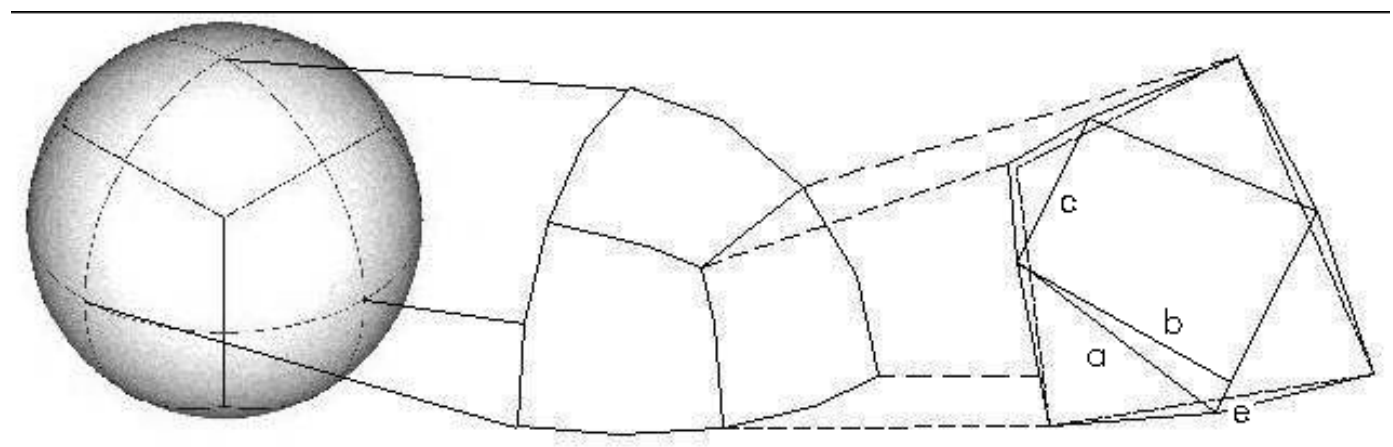

Figura 3: La bola "cometa".

Antes de introducir y describir una serie de modificaciones sobre este diseño (que resultarán en mejoras sustanciales respecto de los factores $S F$ y $R F$ ) haremos una comparación 
entre nuestra propuesta de "cometa" y el "deltoide". Usando los conceptos de $S F$ y $R F$ (Definiciones 2.1 y 2.2, respectivamente), podemos comparar los resultados obtenidos para el caso "deltoide" con el obtenido en nuestro caso, con un diseño mejorado. Así, cuando en el caso "deltoide" $S F=97.2327$ y $R F=90.1386$, en el caso "cometa", $S F=100$ y $R F=98.5341$, lo cual evidencia una mejora absoluta en ambos factores.

En las secciones que siguen resumiremos el procedimiento que proponemos para la construcción de la bola, la cual llevaremos a cabo siguiendo la estrategia de ajustar las dimensiones de los triángulos esquineros $(\S 3)$ y el procedimiento de torsión (o "twist") $(\S 4)$.

\section{$3 \quad$ El $S F$ y el procedimiento de ajuste de triángulos}

El diseño original, definido al comienzo de la $\S 2$, secciona inicialmente el balón de acuerdo a la siguiente distribución (según mostramos en la Figura 1): 24 trapecios, 18 cuadrados y 8 triángulos. Esta distribución ubica un triángulo equilátero en la parte central de cada octante sobre la superficie de la bola. La operación de ajuste de estos triángulos constituye el paso inicial del proceso, y consiste en modificar las dimensiones de cada uno de estos triángulos equiláteros centrales hasta encontrar aquella medida que satisfaga, de una manera eficiente, el hecho de que las longitudes de las circunferencias involucradas sean iguales (o "casi" iguales). Según sea el caso de que se genere un triángulo de menor o de mayor área, este procedimiento logrará acercar o alejar la región triangular de cada octante del balón del centro geométrico del mismo. Debemos observar aquí que, una vez seleccionada una medida para la longitud de la circunferencia $C_{1}$, podemos determinar también todas las medidas de las figuras geométricas que conforman la cobertura del diseño inicial.

A continuación analizaremos brevemente algunas de las estrategias para reajustes de las dimensiones de los triángulos esquineros. Éstas vienen dadas por los casos " $d-a$ ", "d/10", "d/7" y "MAP". Además, consideramos el caso en el que transformamos los cuadrados laterales, adyacentes a cada uno de los triángulos esquineros, en rombos.

En el primer caso, que llamamos " $d-a$ ", si consideramos los cuadrados del diseño inicial y sustraemos el lado $a$ de estos cuadrados de su diagonal $d$, obtendremos una medida que podemos escoger para la longitud del radio de nuestros triángulos esquineros, cual es $d-a$ (ver Figura 4a). Esta cantidad, que nos da una altura para los triángulos equiláteros igual a $\frac{3}{2}(d-a)$ nos recuerda los sencillos, pero ingeniosos cálculos, de los antiguos griegos. Una consecuencia importante de este resultado la constituye el siguiente teorema.

Teorema 3.1 (Pacheco, $2000[3])$ Si $c=\sqrt{3}(d-a)$, entonces

$$
4(a-b)=l\left(C_{1}\right)-l\left(C_{2}\right), \quad \text { y } \quad l\left(C_{3}\right)=1.0006 \% l\left(C_{1}\right) .
$$

Demostración: Dado que $l\left(C_{1}\right)=8 a \sqrt{2}$ y $l\left(C_{2}\right)=(2 a+4 b+4 h+2 a \sqrt{2})$, y como $h=\frac{\sqrt{3}}{2} c=\frac{3}{2}(d-a)$ (por hipótesis) y $d=\sqrt{2} a$, sigue que:

$$
8 a \sqrt{2}-2 a-4 b-4 h-2 a \sqrt{2}=4(a-b) .
$$


Que $l\left(C_{3}\right)$ alcanza el valor especificado sigue de la misma definición de $C_{3}$ y de que $c=$ $\sqrt{3}(d-a)$.

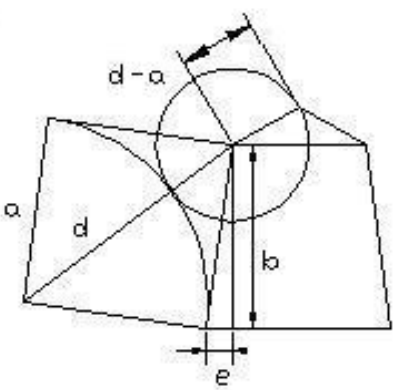

(a)

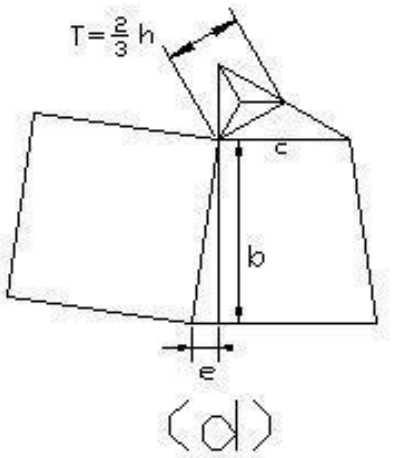

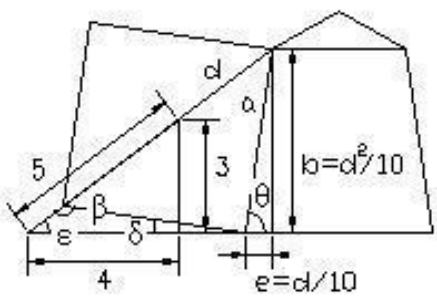

(b)
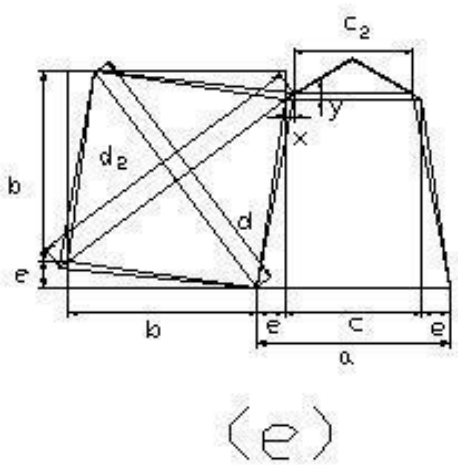
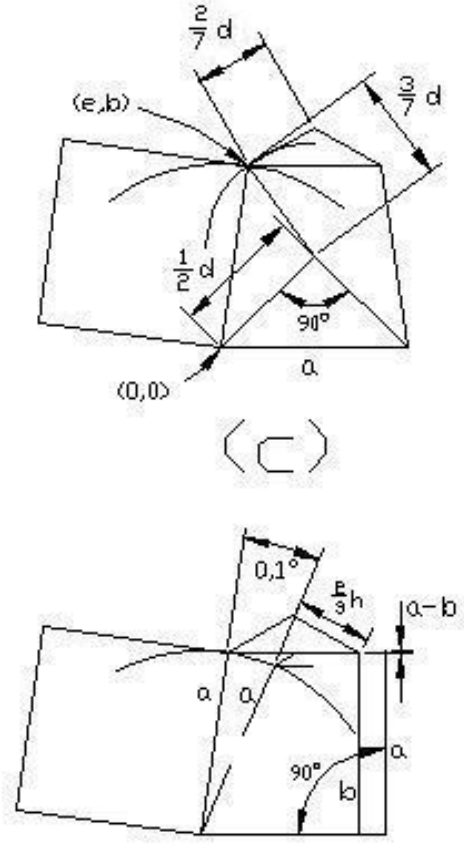

$(f)$

Figura 4: (a) Caso " $d-a$ ". (b) Caso " $d / 10 "$ " (c) Caso " $d / 7$ ". (d) Caso "MAP". (e) Caso "rombo". (f) Los casos (a)-(d) en un arco de $0.1 \mathrm{~mm}$.

Cuando la pendiente de la recta que contienen la diagonal de un cuadrado lateral es igual a $3 / 4$ respecto a la base del trapecio adyacente, nos encontramos con el siguiente interesante resultado que aquí denominamos el caso " $d / 10$ ". Observamos de la Figura $4 b$ que $3 / 4$ es la pendiente de la línea definida por " $d$ ". Para esta pendiente tenemos que $\xi=\arctan (3 / 4)$. De la misma Figura, vemos también que $\beta=\pi-\pi / 4=3 \pi / 4$ y que $\partial=\pi-\beta-\xi$. De donde, $\theta=\pi-\pi / 2-\partial$. Por lo tanto, de $\operatorname{sen}(\theta)=\frac{b}{a} \mathrm{y} \cos (\theta)=\frac{e}{a}$, sigue que, $e=a \cos (\theta)=d / 10$ y $b=a \operatorname{sen}(\theta)=d^{2} / 10$.

En el siguiente caso, que denominamos " $d / 7$ ", buscamos que el valor de $c$ sea tal que, para una circunferencia $C_{1}$ de longitud $l\left(C_{1}\right), \frac{2}{3} h$ aproxima a $\frac{2}{7} d$, con el punto de cruce de las diagonales del trapecio ubicado a una distancia de $\frac{3}{7} d$ desde los extremos de $c$ y de $d / 2$ desde los extremos de la base de longitud $a$ del trapecio. Fijamos como origen de coordenadas un extremo de la base de longitud $a$ del trapecio, y nuestro objetivo será el de encontrar las coordenadas $(x, y)=(e, b)$ correspondientes a uno de los extremos del segmento $c$. Observamos de la Figura 4c, que este punto corresponde al punto intersección de las circunferencias $x^{2}+y^{2}=a^{2}$ y $(x-a / 2)^{2}+(y-a / 2)^{2}=(3 d / 7)^{2}$.

Consideremos ahora el caso " $M A P$ ". Aquí, el lado $T$ del triángulo de apertura, ge- 
nerado a través de aplicar el "twist" (ver $\S 4$ ), lo escogemos de manera tal que su longitud sea igual a $\frac{2}{3} h$, donde $h$ es la altura del triángulo esquinero (definido en el diseño original). Como estamos interesados en que $\frac{2}{3} h$ sea igual al lado $T$ del triángulo "twist", la altura de este triángulo será $\frac{h}{\sqrt{3}}$, con la correspondiente área $A_{T}=\frac{2}{3} h \frac{h}{\sqrt{3}} \frac{1}{2}=\frac{h^{2}}{3 \sqrt{3}}$. Así que, para $T=\frac{2}{3} h, A_{T}=T^{2} \frac{\sqrt{3}}{4}$, y $T=2 \sqrt{\frac{\sqrt{3}}{3} A_{T}}$.

Ahora, mientras mayor sea $c$ (y en consecuencia, $\frac{2}{3} h$ ), menor será el déficit de área y, por lo tanto, también será menor $T$. Por lo que sería interesante buscar y encontrar un punto de "equilibrio" en el cual $T$ sea igual a $\frac{2}{3} h$ (ver Figura $4 d$ ). Encontramos este punto geométrico al implantar un algoritmo que nos permitió estimar $c$ tal que $T-\frac{2}{3} h=0$, y a partir del cual también pudimos estimar las coordenadas del nuevo punto (ver Tabla 1).

Una segunda opción por considerar, con la que alcanzamos un efecto similar a cuando modificamos las dimensiones de los triángulos esquineros, consiste en alterar las medidas de los tres cuadrados laterales que rodean cada uno de los triángulos esquineros. En este proceso de ajuste, y que aquí denominamos el caso "rombo", dejamos fijas las dimensiones de los cuadrados frontales. Con este procedimiento logramos el mismo efecto de acercar o alejar el área central de cada octante al centro de la esfera, con el añadido de que en este caso los tres cuadrados laterales que rodean a cada uno de los ocho triángulos los transformamos en rombos (ver Figura 4e). La estrategia a seguir cuando aplicamos este caso es la siguiente: Primeramente, hacemos $l\left(C_{1}\right)=l\left(C_{3}\right)$ para obtener el valor de $c$ (ver Tabla 1, columna 4); a continuación, convertimos los cuadrados laterales en rombos; esto lo hacemos extendiendo una de sus diagonales, la correspondiente a $C_{2}$, dejando la correspondiente a $C_{1}$ fija (ver Tabla 1 , columna 6 ).

Para facilitar los cálculos fijaremos como origen de coordenadas un vértice del triángulo esquinero. Nuestro objetivo es encontrar un punto en el interior del triángulo esquinero, de coordenadas $(x, y)$, sobre la diagonal extendida que nos permitirá alcanzar el efecto deseado de esfericidad (ver la geometría involucrada en la Figura 4e). Para ello, requerimos definir unos parámetros adicionales, llamémosles $b_{2}, c_{2}$ y $d_{2}$, tales que $\tan (\theta)=\frac{y}{x}=\frac{b-e}{b+e}=m$, donde $y=b_{2}-b$ y $x=\frac{c}{2}-\frac{c_{2}}{2}$, de aquí que $y=m x, b_{2}=y+b$ y $c_{2}=c-2 x$. También vemos que $d_{2}=d+2 \sqrt{x+y}=\sqrt{2} a+2 \sqrt{x+y}$. Además, como $h=\frac{\sqrt{3}}{2} c$, también definimos $h_{2}=\frac{\sqrt{3}}{2} c_{2}=\frac{\sqrt{3}}{2}(c-2 x)$. Ahora, haciendo $l\left(C_{2}\right)=l\left(C_{1}\right)$, tenemos que $l\left(C_{2}\right)=2 d_{2}+2 a+4 b_{2}+4 h_{2}=8 d=l\left(C_{1}\right)$, o bien, $d_{2}+a+2 b_{2}+2 h_{2}=\frac{l\left(C_{1}\right)}{2}$. Reemplazando las correspondientes expresiones para $d_{2}, b_{2}$ y $h_{2}$, junto con la de $y=m x$, vemos que $d+2 \sqrt{x^{2}+m^{2} x^{2}}+\frac{d}{\sqrt{2}}+2(b+m x)+\sqrt{3}(c-2 x)=\frac{l\left(C_{1}\right)}{2}$. Después de algunas manipulaciones algebraicas adicionales, y haciendo, por ejemplo $l\left(C_{1}\right)=56 \mathrm{~cm}$, llegamos a que: $x=\frac{21 \sqrt{2}-7-\sqrt{6} c-2 \sqrt{2} b}{2 \sqrt{2}\left(\sqrt{1+m^{2}}+m-\sqrt{3}\right)}$, donde $m=\frac{b-e}{b+e}$. Por lo que también $y=\frac{b-e}{b+e} x$. Y de donde podemos fácilmente estimar el nuevo valor para $c: c_{2}=c-2 x$.

Lo cual demuestra que al aplicar este procedimiento de transformación de los cuadrados en rombos se acorta la longitud del lado del triángulo esquinero (ya que de $c$, pasa ahora a ser $\left.c_{2}\right)$, a la vez que se cumple con que $l\left(C_{1}\right)=l\left(C_{2}\right)=l\left(C_{3}\right)$, como queríamos. 


\begin{tabular}{c|cccccc}
\hline \hline & $d-a$ & $d / 10$ & $M A P$ & "C1C3" & $d / 7$ & "rombo" \\
\hline$c$ & 3.5511 & 3.5497 & 3.5422 & 3.5406 & 3.5349 & 3.0920 \\
$b$ & 4.9001 & 4.9 & 4.8995 & 4.8993 & 4.8989 & 5.0672 \\
$e$ & 0.6993 & 0.7 & 0.7038 & 0.7046 & 0.7074 & 0.9289 \\
$\frac{2}{3} h$ & 2.0503 & 2.0494 & 2.0451 & 2.0441 & 2.0409 & 1.7852 \\
$T$ & 1.9867 & 1.9959 & 2.0451 & 2.0553 & 2.0908 & 1.8281 \\
$R F \%$ & 100.0890 & 100.0752 & 100.0000 & 99.9842 & 99.9284 & 99.9462 \\
\hline \hline
\end{tabular}

Tabla 1: Tabla comparativa en el procedimiento de ajuste de triángulos.

\subsection{Conclusiones del procedimiento de ajuste de triángulos}

En el proceso de ajuste de los triángulos esquineros para resolver el problema de la esfericidad, hemos estudiado cinco casos en los que hemos encontrado valores diferentes para $c$, que no difieren mucho entre sí, consecuencia de lo cual, aunque tengamos triángulos esquineros con áreas diferentes, las mismas también son "próximas" entre sí, lo que determina, con un error absoluto, relativamente pequeño, que $l\left(C_{1}\right) \approx l\left(C_{2}\right) \approx l\left(C_{3}\right)$. Otro interesante resultado relativo a las coordenadas halladas en los diferentes casos es el que, además de estar relativamente "cerca" unas de otras, también pertenecen a una misma circunferencia de radio $a$.

Es importante observar aquí que los casos denominados " $d-a$ ", " $d / 10$ ", " $d / 7$ ", "MAP" y "C1C3" (que definen distintos valores para $c$ ) generan coordenadas $(x, y)=(e, b)$ que caen sobre una misma circunferencia de radio $a$, con centro en uno de los vértices de la base $a$ del trapecio adyacente al triángulo esquinero. Más aún, estas coordenadas pertenecen a un arco de longitud aproximada igual a $(a-b) / 5$. Así, por ejemplo, si $l\left(C_{1}\right)=56 \mathrm{~cm}$, la longitud de arco resultará ser menor a la correspondiente de una décima de grado (esto es, aproximadamente igual a $0.1 \mathrm{~mm}$, Figura $4 \mathrm{f}$ ).

En la Tabla 1 mostramos un resumen comparativo de los diferentes casos analizados en el procedimiento de ajuste de triángulos esquineros y cuadrados laterales. En este proceso buscamos obtener factores de esfericidad iguales a 1 (o, por lo menos, "muy cercanos" a 1). La tabla muestra los valores numéricos, todos redondeados a cuatro decimales, correspondientes a los parámetros que hemos considerado en los diferentes casos analizados para $l\left(C_{1}\right)=56 \mathrm{~cm}$. A excepción de la última columna de la Tabla 1 , que difiere drásticamente del resto de los valores debido a la transformaciones de los cuadrados en rombos, hemos ordenado las columnas respecto a $c$, de mayor a menor. Observemos que, al incrementarse $c$, disminuyen $b$ y $\frac{2}{3} h$ y aumentan $e$ y $T$. Resulta interesante también observar que los dos casos " $d / 10$ " y " $d / 7$ " involucran estrategias simples y números enteros en sus medidas, además de que tienen un error absoluto en sus parámetros, con respecto al caso " $M A P$ " del orden de $10^{-2}$.

Resulta importante observar que, aunque estas estrategias nos permiten alcanzar un factor de esfericidad de 1 (inmejorable), el factor de redondez, $R F$, sigue mostrando un déficit. Afortunadamente, proponemos a continuación una estrategia que nos permitirá resolver esta dificultad, a la vez que obtendremos un nuevo tipo de bola, la bola "twist", con la que alcanzamos un factor $R F$ igual a 1. 


\section{El $R F$ y el problema del faltante de área $(M A P)$}

Buscamos ahora eliminar los puntos de tensión y hundimiento de nuestro diseño anterior a través de la aplicación de un procedimiento de torsión (o "twist"), el cual logra agregar a la cubierta de la bola un área adicional y así mejorar el factor de redondez $R F$, sin causar mayor alteración del factor de esfericidad $S F$.

\subsection{Procedimiento de torsión o "twist"}

El segundo paso del procedimiento que aquí proponemos en el diseño de la estructura de la cobertura del balón, está dirigido a resolver el problema del faltante de área (o el "Missing Area Problem", o $M A P$ ). El $M A P$ se define como el número positivo que es igual al valor absoluto de la diferencia de superficies de dos áreas, el área real (obtenida a partir de fijar la longitud de una circunferencia de radio igual al de la esfera) y el área correspondiente a la suma de las áreas de todos los paneles definidos en la superficie de la bola.

El MAP lo resolvemos por considerar lo que denominamos aquí el "twist", el cual consiste, básicamente, en rotar seis áreas de la superficie esférica, cada una de las cuales está compuesta por cuatro "cometas" (Figura 5(b)). A través de la aplicación del "twist" se generan unos pequeños triángulos en las ocho "esquinas" del sólido, o puntos "Y" (que corresponden a los puntos intersección de exactamente tres de las "cometas"). De manera que, a partir de esta operación de rotación, se dará lugar a la creación de un área adicional en ocho puntos de la superficie esférica, exactamente en los centros de los octantes de la esfera. Con esa operación se logra generar, precisamente, un área que se añade a la superficie del sólido, sin que el mismo pierda su esfericidad natural. El efecto logrado será una evidente mejora en el $R F$.
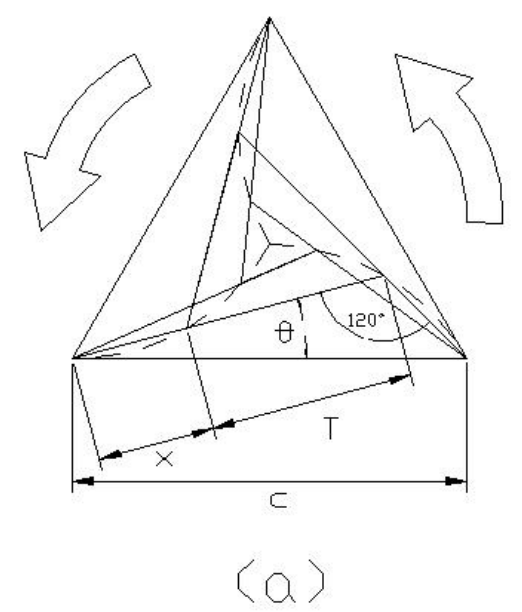

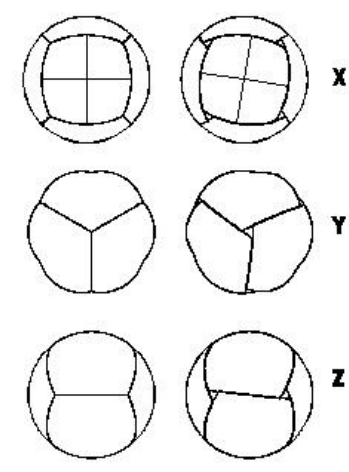

(b)

Figura 5: (a) El "twist". (b) La bola "twist".

La Figura 5a muestra una "simulación" del proceso de "twist", que genera un triángulo de apertura en el centro del triángulo esquinero. A partir de la geometría observada en 
la Figura, podemos obtener algunas relaciones matemáticas entre el ángulo de rotación y el área de apertura generada por el procedimiento "twist". Por la fórmula de Herón, el área del triángulo esquinero de lado $c$, es $A r e a_{e s q}=\frac{c^{2}}{2^{2}} \sqrt{3}$. Por otra parte, y aplicando nuevamente la fórmula de Herón, el área de cada uno de los tres triángulos de lado $c^{\prime}+x$, es Area $_{c^{\prime}+x}=\frac{1}{2^{2}} \sqrt{\left[\left(2 x+c^{\prime}\right)^{2}-c^{2}\right]\left[c^{2}-\left(c^{\prime}\right)^{2}\right]}$. Por lo que el área del triángulo de apertura es $A r e a_{a p}=$ Area $_{e q q}-3 A r e a_{c^{\prime}+x}$. De donde,

$$
x=\frac{1}{2}\left(\sqrt{\frac{2^{4} A r e a_{c^{\prime}+x}^{2}}{c^{2}-\left(c^{\prime}\right)^{2}}+c^{2}}-c^{\prime}\right),
$$

con Area $_{c^{\prime}+x}=\frac{\text { Area }_{e s q}-\text { Area }_{a p}}{3}$.

Observemos también que si denotamos por difarea el error absoluto del área (cuando aproximamos el área real por el área obtenida al sumar las áreas de todos los paneles, sin "twist"), tenemos que $A r e a_{a p}=\frac{\text { difarea }}{8}$ (pues son ocho los triángulos de apertura), que sería el área ideal a considerar para el triángulo de apertura, el cual a su vez, determina el ángulo de rotación necesario en el procedimiento "twist".

Ahora, si denotamos por $\theta$ el ángulo de rotación (ver Figura 5a), del teorema del seno, sigue que

$$
\theta=\arcsin \left(\frac{x \sin (2 \pi / 3)}{c}\right)
$$

Este resultado demuestra la relación existente entre el ángulo de rotación, inducido por aplicar el "twist", $\theta$, y el lado $x$, el cual varía en longitud según se va generando el triángulo de apertura. Así, por ejemplo, cuando $\theta=0, x=0$, y cuando $\theta=\pi / 6, c^{\prime}=0$ y $x=c / \sqrt{3}$.

Por otra parte, la apertura de un triángulo en el centro del triángulo esquinero genera tres trayectorias en espiral (Figura 5a), las cuales pueden pensarse como trayectorias que parten del centro del triángulo y llegan hasta los vértices del triángulo esquinero (o viceversa). La ecuación en coordenadas polares, para cada una de estas espirales, la podemos deducir del modelo matemático

$$
\left\{\begin{array}{l}
r^{\prime}(\theta)=k r, \quad \frac{\pi}{6} \geq \theta>0, \quad k<0, \\
r\left(\frac{\pi}{6}\right)=\frac{2}{3} h,
\end{array}\right.
$$

donde $h$ es la altura del triángulo esquinero. Es éste un problema de valores iniciales (PVI) de solución conocida y que, en este caso, representa la relación de proporcionalidad existente entre la variación de la longitud $r$ del radio (i.e., $x+c^{\prime}$ ) con respecto al ángulo de rotación y el mismo radio $r$. El problema (3) es fácil de resolver y tiene la solución

$$
r=\frac{2}{3} h e^{k(\theta-\pi / 6)}, \quad \text { con } k<0 \text {. }
$$

Así, si $\theta=\pi / 6$, entonces $r=\frac{2}{3} h$, y si $\theta=0, r=c$, con $k=\frac{-6 \ln (3 c / 2 h)}{\pi}$ (para el caso en que $\left.c=\sqrt{3}(d-a), k=-\frac{6}{\pi} \ln \sqrt{3}\right)$.

Es decir, el ángulo de rotación del "twist" debe ser el correspondiente a nuestras necesidades de faltante de área. Una vez encontrada el área requerida para cada triángulo 
(equilátero) de apertura, podemos determinar su lado que, junto con la aplicación de (1), (2) y (4), nos permitirá calcular el ángulo apropiado para el "twist", lo que a su vez justifica la creación de los triángulos de apertura, y resuelve el MAP. Recíprocamente, dado el ángulo de rotación $\theta$ podemos también determinar, a partir de (2) y (4), los valores de $x, r$ y $c^{\prime}$ (el lado del triángulo de apertura), y por ende, podemos también calcular el área del triángulo de apertura.

Si bien, el ángulo máximo de rotación podría ser de $\pi / 4$ radianes, en la práctica éste es mucho menor, y para el caso de la "cometa", por ejemplo, requerimos tan sólo de una rotación aproximada de unos $\pi / 22.5$ radianes $^{1}$. Según sea el área del triángulo seleccionado en el primer paso ( $(3)$, se producirá el correspondiente $M A P$ que, a su vez, será resuelto mediante el segundo paso del proceso, cuando aplicamos el "twist" apropiado. Así, para dimensiones de triángulos relativamente pequeñas, obtenidas en el primer paso, el grado del "twist" necesario será mayor, y para medidas de triángulos grandes, el grado del "twist" será menor. De manera que existe una relación inversamente proporcional entre el área del triángulo equilátero, definido en el primer paso, y el área del triángulo de apertura dada por el "twist", en el segundo paso.

Por la dinámica y la continuidad inherentes al proceso descrito (i.e., la relación de continuidad existente entre las dimensiones de los triángulos de apertura y las dimensiones de los triángulos esquineros), resulta claro que existirá un "punto ideal", donde el lado del triángulo de apertura coincidirá con el radio del triángulo esquinero obtenido en el paso 1 (i.e., el caso " $T=\frac{2}{3} h "$ ), y para el cual $S F \approx 1$.

\subsection{Resumen de resultados para el $M A P$}

La combinación ideal entre el área de un triángulo, definida por el paso 1, y el ángulo determinado por el "twist" en el paso 2, va a depender de cuán exacto queremos que sea el $S F$ antes de utilizar el "twist", para mejorar el $R F$. No necesariamente en todos los casos $S F$ será igual 1 (para luego mejorar el factor $R F$ ).

En la Tabla 1 mostramos, para cada uno de los casos considerados en la $\S 3$, cómo queda el $R F$ después de realizar la operación de "twist". Aquí tomamos como referencia el caso $T=\frac{2}{3} h(R F=100 \%)$. Es interesante observar el hecho de que los requerimientos del faltante de área son, en efecto, compensados con el área creada al aplicar el "twist" y generar los triángulos de apertura.

Según hemos corroborado, para diferentes valores de $l(C)$, obtenemos las soluciones más eficientes cuando el lado del triángulo de apertura tiene una longitud aproximada de $\frac{l(C)}{8}-\frac{l(C)}{8} \frac{1}{\sqrt{2}}($ i.e., $d-a)$, medida correspondiente a un "twist" de $\pi / 22.5$ radianes, como más arriba señalamos.

\footnotetext{
${ }^{1}$ El cálculo del "twist" se puede realizar al establecer que cada conjunto de cuatro deltoides adyacentes (i.e., 1/6 de la superficie del balón) giran con respecto a su centro geométrico, sobre una circunferencia de radio igual a $r=a / 2+1 / 3 h+b$, que se extiende desde este centro hasta la esquina $Y$. Si queremos que el desplazamiento o torsión de las esquinas $Y$ después del "twist" sea igual $x=T / \sqrt{(} 3$ ) (i.e., el radio del triángulo de apertura), entonces el ángulo de rotación se puede obtener, de manera aproximada, por $x / r$ que, para el caso arriba señalado, equivale aproximadamente a $\pi / 22.5$ radianes (ver [4] para detalles).
} 


\section{Comentarios finales}

La literatura especializada acerca del tema es escasa y prácticamente inexistente. Si bien, en la práctica, no existe una "bola ideal", en este trabajo proponemos un modelo para la construcción y el diseño de una bola que verifica propiedades y parámetros esenciales de esfericidad y redondez, que caracterizan a una "bola ideal". Otros modelos conocidos no cumplen, sino de una manera muy aproximada, con estas propiedades o parámetros de medición.

\section{Referencias}

[1] Clinton, J.D. (2002) "A limited and biased view of historical insights for tessellating sphere", in: G.A.R. Parke \& P. Disney (Eds.) Procedings of the Fifth International Conference on Space Structures, Thomas Telford Publishing, Guildford, UK: 423-432.

[2] Ghyka, M. (1977) The Geometry of Art and Life. Dover Publications, Inc., New York.

[3] Pacheco, F. (2002) "Cover for a ball or sphere", WO 02/30522. PCT Gazzette. Issue $16 / 2002$.

[4] Pacheco, F. (2004) "Ball having improved roundness and sphericity", Patent Cooperation Treaty. Appl. PCT/CR04/00001.

[5] Shaper, H. (1997) "Inflatable game ball", US PAT. 5,674,149.

[6] Smith, T. (2000) "Penrose tilings and Wang tilings", http://www.innerx .net/personal/tsmith/pwtile.html.

[7] Wang, H. (1961) "Proving theorems by pattern recognition. II", Bell Systems Tech. J., 40: 1-41.

[8] Weisstein, E.W. (1999) "Archimedean solids", http://mathworld.wolfram .com/ArchimedeanSolid.html. 